EPSC Abstracts

Vol. 14, EPSC2020-226, 2020, updated on 28 Nov 2020

https://doi.org/10.5194/epsc2020-226

Europlanet Science Congress 2020

(C) Author(s) 2020. This work is distributed under

the Creative Commons Attribution 4.0 License.

\title{
Sub-pixel geometric modeling and spectral fitting for Rosetta/VIRTIS-M measurements of the nucleus of comet 67P
}

\author{
David Kappel ${ }^{1,2}$, Gabriele Arnold ${ }^{2}$, Liubov Moroz ${ }^{2}$, Andrea Raponi ${ }^{3}$, Mauro Ciarniello ${ }^{3}$, Federico Tosi $^{3}$, \\ Stephane Erard ${ }^{4}$, Batiste Rousseau ${ }^{3}$, Cedric Leyrat ${ }^{4}$, Gianrico Filacchione ${ }^{3}$, and Fabrizio Capaccioni ${ }^{3}$ \\ ${ }^{1}$ Institute of Physics and Astronomy, University of Potsdam, Potsdam-Golm, Germany (dakappel@uni-potsdam.de) \\ ${ }^{2}$ Institute of Planetary Research, German Aerospace Center (DLR), Berlin, Germany \\ ${ }^{3}$ INAF-IAPS, Rome, Italy \\ ${ }^{4}$ LESIA, Observatoire de Paris, Université PSL, CNRS, Sorbonne Université, Université de Paris, France
}

\begin{abstract}
We aim at retrieving physical and compositional surface properties of the nucleus of comet 67P/Churyumov-Gerasimenko (hereafter 67P) from VIS-IR hyperspectral images ('cubes'). Here we report on our progress in the geometric modeling and spectral fitting.
\end{abstract}

\section{Introduction}

The measured cubes have been acquired with VIRTIS-M instrument [1] aboard Rosetta. Starting from a digital shape model of 67P [2], the radiance measured by a pixel results from sub-pixel radiance contributions of several shape model facets weighted by the wavelength-dependent spatial point spread function (PSF) of the instrument. Based on a corresponding sub-pixel geometric modeling, we have computed the weighting coefficients and quantified the PSF [3]. The radiance contribution from a single facet can be simulated from physical and compositional parameters defined on this facet, using a photometric model (Hapke or Shkuratov [4,5]). Besides facilitating the consideration of PSF effects, this approach allows us to better approximate the rugged fine-scale topography of 67P that leads to varying observation/illumination/shadowing conditions on sub-pixel scales. Also, the retrieval of parameters common to multiple acquisitions requires a definition of surface parameters bound to the shape model instead of the respective pixel footprints. In this work, we focus on the spectral fitting of entire sets of cubes.

\section{Retrieval algorithm}

The Bayesian Multi-Spectrum Retrieval algorithm MSR [6] fits the synthetic spectra to the measured ones by iteratively varying the facet properties. MSR takes into account constraints and Bayesian a priori information on the facet properties (mean values, standard deviations, correlation lengths/times/wavelengths) as well as measurement error information. Moreover, consistency requirements are respected. For instance, for measurements acquired at similar times, there are facet properties (surface roughness, particle size, composition, etc.) that do not change between repeated observations and can be treated and retrieved as common to those observations. To achieve this, MSR regards many measurements of a selected surface region under different illumination and observation conditions, shadowing state, spatial resolution, and at different 
wavelengths as a single meta-measurement. This is analogous to the meta-measurement formed by measurements at different single wavelengths, called a spectrum.

\section{Preliminary results}

At the present stage, MSR is tested to retrieve maps of shape model facet properties from a metameasurement encompassing tens of VIRTIS-M-cubes, corresponding to the order of a million measured spectra, see Fig. 1.

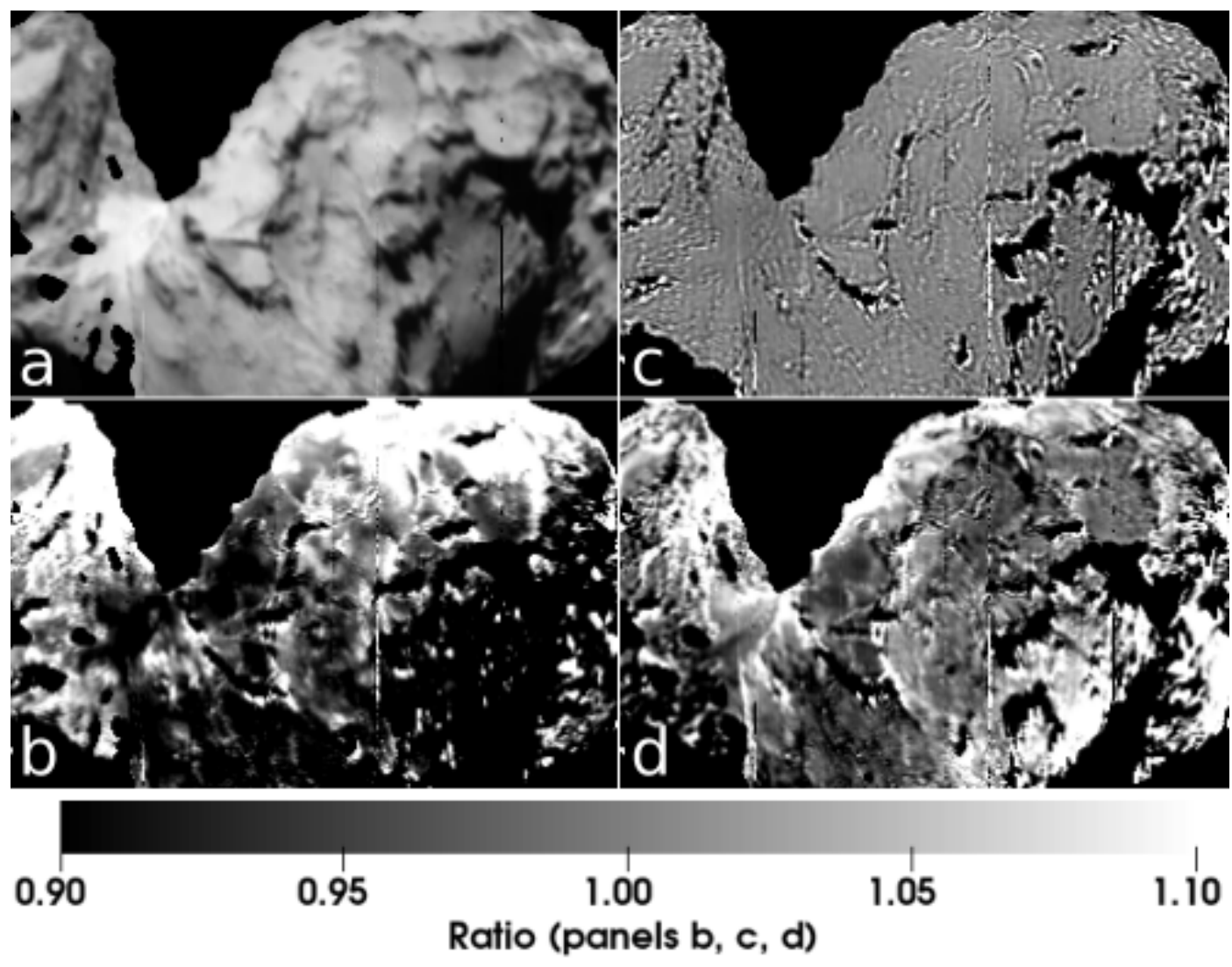

Fig. 1. (a) measured image at $1 \mu \mathrm{m}$ (VIRTIS-M acquisition I1 00367585390 , heliocentric distance $\sim 3.5 \mathrm{AU}$ ); (b) scaled ratio of Akimov disk function of the scene and measured image; (c) ratio of best fit and measurement from fitting just this single cube using the Hapke model; (d) ratio of preliminary best fit and measurement from simultaneously fitting this and 24 other cubes using the Hapke model.

Panel (a) shows a calibrated and pre-processed measured VIRTIS-M cube, represented at $1 \mu \mathrm{m}$, mainly showing 67P's northern hemisphere.

Panel (b) illustrates residual variations when the first-order effect of the complex topography of 67P is removed using the Akimov disk function [5], which describes the photometry of utterly rough surfaces. These residual variations can be due to limits in the applicability of the Akimov disk function or real physical variations in texture or composition. The phase angle $\left(\sim 40^{\circ}\right)$ is too large for substantial opposition effects to show up.

In the case where we fit the entire cube using the Hapke model, panel (c) shows very little residual variations that are mainly associated with local terminators and slight PSF model imprecisions. This 
image illustrates that our present setup allows us to fit the measurements very accurately when the facet properties can vary freely within the frame of our Bayesian regularization for one cube. For this comparison, we selected as free parameters the single-scattering albedo and phase function asymmetry parameter spectra for two intimately mixed endmembers as properties that are constant over all facets, and the relative abundance of the two endmembers along with the roughness angle and filling factor as properties that can vary between facets.

Finally, panel (d) exhibits moderate residual variations. Here, we fitted a meta-measurement of 25 cubes at various illumination and observation conditions and displayed the one also represented in the other panels. The fitting took about one week on a desktop computer. Now the facet properties cannot vary as freely as for case (c), because simultaneously they also have to be compatible with all other considered cubes. The retrieved facet properties are therefore more well-grounded candidates for the actual surface properties. This panel demonstrates that using information from many hyperspectral images helps to reduce overfitting. We also note that the here utilized set of free parameters is not able to fully capture the spectral variability of the measurements within the frame of this model, pointing to the necessity of additional free parameters, or difficulties of the Hapke model to simultaneously parameterize the different cubes in a consistent way.

At this stage, it is not clear yet, how the retrieved facet properties are related to actual surface properties of 67P. At the meeting we will present different parameter sets that lead to equally well fits and discuss their plausibility. A similar investigation based on the Shkuratov model as well as a detailed error analysis, based on synthetic VIRTIS-M cubes, to investigate interferences between the retrieved and other parameters, are the next steps in this ongoing work. Finally, we expect our approach to be capable of identifying local surface property variations in a physically and mathematically well-grounded way, and we will investigate their correlations with morphologic regions on 67P.

\section{Acknowledgements}

We thank the following institutions and agencies for support of this work: Italian Space Agency (ASI, Italy). Centre National d'Etudes Spatiales (CNES, France), DLR (Germany). D.K. acknowledges DFGgrant KA 3757/2-1.

\section{References}

[1] Coradini et al. (2007) Space Sci. Rev. 128, 529. [2] Preusker et al. (2017) A\&A 607, L1. [3] Kappel et al. (2019) EPSC-DPS 2019, EPSC-DPS2019-456. [4] Hapke (2012) Theory of Reflectance and Emittance Spectroscopy, 2nd edn. (Cambridge University Press). [5] Shkuratov et al. (2011) Planet. Space Sci. 59, 1326. [6] Kappel (2014) J. Quant. Spectrosc. Rad. 133, 153. 